\title{
Comparative Analysis of B-Mode Breast Ultrasound Image Enhancement Techniques
}

\author{
Madan Lal \\ Department of Computer Engineering \\ Punjabi University \\ Patiala
}

\author{
Lakhwinder Kaur \\ Department of Computer Engineering \\ Punjabi University \\ Patiala
}

\begin{abstract}
Acquisition of ultrasound images is cheap and noninvasive as it does not require ionizing radiations as compared to other medical imaging techniques but the problem with these images lies in its inherent characteristics like speckle noise and low contrast. In this paper the performance of various image enhancement techniques are compared by applying them on B-Mode breast ultrasound images (BUS) and by using the essential quantitative metrics like signal to noise ratio (SNR), Edge Preserving Index (EPI) and Structured Similarity Index (SSIM).
\end{abstract}

\section{Keywords}

Speckle Noise, B-Mode breast ultrasound (BUS) image, SNR, EPI, and SSIM.

\section{INTRODUCTION}

Ultrasound medical imaging uses high frequency sound waves to visualize body's internal structures and create pictures of the tissues and organs [1]. As the sound waves pass through a body, they are reflected back in different ways, depending on the characteristics of the tissues encountered. Among the currently available medical imaging techniques, ultrasound imaging is regarded as a noninvasive, practically harmless, portable, accurate, and cost effective method of diagnosis [2]. These properties make the ultrasound imaging be the most prevalent diagnostic tool to detect abnormalities in the soft tissues of human bodies. Like all imaging modalities ultrasound images are still subject to inherit artifacts like low contrast and speckle which arise from coherent wave interferences. Speckle reduces image contrast, detailed resolution and makes it difficult to identify abnormality pattern which may indicate disease. Various spatial [3-5], [911] and frequency domain techniques [12] have been developed to reduce the speckle noise. No matter which method is used to enhance the BUS image, it should preserve radiometric information, edge information and last but not least, spatial resolution. In this paper some of these techniques are discussed and their comparative analysis is carried out.

\section{CONTRAST ENHANCEMENT}

Histogram equalization is one of the well known image enhancement technique [3]. It flattens and stretches the dynamic range of image histogram and resulting in overall contrast improvement. Histogram equalization assign the intensity value of pixels in the input image such that output image contain a uniform distribution of intensities [4]. This technique can be applied to whole image or on the subparts of an image [4] [6]. In spite of its fundamental advantage histogram equalization has a major drawback of changing the brightness globally which results in either under or over saturation of important regions [6].

\section{SPECKLE REDUCTION}

Main problem of BUS images is the presence of speckle noise. Over the years numerous despeckle techniques have been proposed, aiming at improving the separation of targets from background with minimum data loss. These techniques can be divided into following categories.

i) First category of filters [3] [5] smoothens the central pixel on the basis of neighborhood pixel statistical values like mean, median etc.

ii) Second types of filters [7-9] are those filters which smoothen the most homogeneous neighborhood regions around each pixel and leave the edges. These methods tend to reduce the speckle noise while preserving important diagnostic information in an image.

iii) Third category filters [10-11] use partial differential equations to remove the speckle. These filters use a Sigmoidal function rather than a step function.

iv) Forth category includes multi scale methods which use wavelets for speckle removal [12] [15] [17].

This paper evaluates the performance of contrast enhancement method along with speckle filtering methods including spatial adaptive filtering methods, diffusion filtering methods and wavelet based method by enhancing B-Mode BUS images.

\subsection{Mean Filter}

The mean filter [3] [16] is a spatial sliding window filter that replaces the center pixel value with average of all pixel values in the window. The window used in mean filter is generally square but it can have any shape. This is the least satisfactory method of speckle reduction as it results in loss of detail and resolution. It can be used for applications where resolution is not the first concern.

\subsection{Median Filter}

In median filter, [3] the central pixel value in the window is replaced with median value of neighbors in the window. The median filter [5] [16] is able to decrease random impulsive noise without blurring edges. Median filter produces less blurred images but its disadvantage is that it takes extra time in sorting the neighborhood values in order to find the median of a small neighborhood.

\subsection{Lee Filter}

Lee filter [7] and [8] is designed based on multiplicative speckle model. It eliminates speckle noise while preserving edges and features. Here smoothing is performed only if the variance over an area is low and no smoothing is performed on high variance area, which is near edges. For uniform regions it produces output values close to the local mean and for high contrast regions it produces values close to the 
original input. Limitation of Lee filter is that it ignores the noise near edges and lines.

\subsection{Frost Filter}

The Frost filter [9] is an adaptive and exponentially weighted mean filter. It replaces the pixel of interest with a weighted sum of the values within the moving kernel. The weighting factor decrease with distance from the pixel of interest and it increase with the increase in variance within the kernel. It makes a balance between averaging and all pass filters.

\subsection{Wiener Filter}

Weiner filter [3] [16] reduce the amount of noise in a signal by comparing received signal with estimation of desired noiseless signal. It performs smoothing of image based on the computation of local image variance. When the local variance of image is small it performs more smoothing and when the local variance is large it performs less smoothing. Since it needs more computation time than linear filters still it is able to preserve edges and other high frequency components of the image.

\subsection{Wavelet Based Filter}

Wavelets are mathematical functions which divide the input data into different frequency components [12]. The noise reduction process in wavelet domain is called wavelet thresholding. In wavelet thresholding input image is decomposed into approximation and detailed sub-bands and then soft or hard thresholding is applied. The selection of threshold plays an important role in noise reduction.

\subsection{Speckle Reducing Anisotropic Diffusion}

Speckle reducing anisotropic diffusion [11] is also a nonlinear anisotropic diffusion method which iteratively processes the noisy image with adaptive weighted filters, reduce noise and preserve edges. $\mathrm{Yu}$ and Acton [11] proposed diffusion constant which is in term of "instantaneous coefficient of variation" as a function of local gradient magnitude and laplacian operator. Here Lee filter and Frost filter are casted into framework of diffusion method.

\subsection{Anisotropic Diffusion}

Anisotropic diffusion [10] is a nonlinear technique which simultaneously performs contrast enhancement and noise reduction. This filter removes the noise from an image by using partial differential equation. Smoothing is carried out depending on the edges in the image and their directions. It smoothes homogeneous regions but preserve the edges.

\section{PERFORAMNE METRICS}

The measurement of ultrasound image enrichment is not easy as there is no unique algorithm available to measure the enhancement. Some statistical tools are used to measure the enrichment. The mathematically defined image quality measures [13-14] [17] include Signal-to-Noise Ratio (SNR), Edge Preservation Index (EPI) and structured similarity index (SSIM).The image quality measures predicted between the original image $\mathrm{f}(\mathrm{m}, \mathrm{n})$, and the reconstructed image $\mathrm{g}(\mathrm{m}, \mathrm{n})$ are given below.

\subsection{Signal to Noise Ratio (SNR)}

Signal to Noise Ratio (SNR) is a subjective qualitative measurement of distortion. It is calculated as:

$$
\mathrm{SNR}=10 \log _{10} \frac{\sigma_{\mathrm{g}}^{2}}{\sigma_{\mathrm{e}}^{2}}
$$

Where $\sigma_{\mathrm{g}}^{2}$ is variance of noise free original image and $\sigma_{\mathrm{e}}^{2}$ is the variance of error. The value of SNR should be high for good quality image.

\subsection{Edge Preservation Index (EPI)}

The filters edge preserving ability is compared by EPI [17]. It is calculated as:

$$
\mathrm{EPI}=\frac{\sum(\Delta \mathrm{I}-\overline{\Delta \mathrm{I}}) \sum(\Delta \mathrm{F}-\overline{\Delta \mathrm{F}})}{\sqrt{\sum(\Delta \mathrm{I}-\overline{\Delta \mathrm{I}})^{2} \sum(\Delta \mathrm{F}-\overline{\Delta \mathrm{F}})^{2}}}
$$

Where $\Delta \mathrm{I}$ and $\Delta \mathrm{F}$ are high pass filtered versions of image I and $\mathrm{F}$, obtained with a $3 \times 3$ pixel standard approximation of Laplacian operator. The larger value of EPI means more ability to preserve edges.

\subsection{Structural Similarity Index (SSIM)}

Structural similarity index is used to measure similarity between two images. The measure between two images $\mathrm{X}$ and $\mathrm{Y}$ of common size is calculated as:

$$
\operatorname{SSIM}=\frac{\left(2 \mu_{X} \mu_{Y}+2.55\right)\left(2 \sigma_{X Y}+7.65\right)}{\left(\mu_{X}^{2}+\mu_{Y}^{2}+2.55\right)\left(\sigma_{X}^{2}+\sigma_{Y}^{2}+7.65\right)}
$$

Where $\mu \mathrm{x}, \mu \mathrm{y}$ are mean of $\mathrm{X}$ and $\mathrm{Y} . \sigma 2 \mathrm{x}$ is variance of $\mathrm{X}$ and $\sigma 2 \mathrm{y}$ is variance of $\mathrm{Y}$. $\sigma \mathrm{xy}$ is the covariance of $\mathrm{X}$ and $\mathrm{Y}$. The result of SSIM lies between -1 and 1 .

\section{EXPERIMENT RESULTS AND ANALYSIS}

For quantitative analysis, Speckle was simulated on Test image [15] Synth.tif using speckle simulator given by Aleksandra Pizurica et al. [15]. Experiments were conducted on speckle simulated synthetic image by adding speckle noise of different values. Image enhancement methods were implemented using image processing toolbox of MATLAB. To quantify the performance gain three quality metrics, Signal to Noise Ratio, Edge Preservation Index and Structural Similarity Index were used. The output values for different parameters were recorded for different methods in Table 1. For implementation of kernel based filters (Mean, Median, Lee and Wiener) uniform window size [5 5] was used, as performance of these filters changes with change in window size. For implementation of wavelet filter "db12" up to level 5 was used. As diffusion based filters are applied repeatedly, so number of iterations used for both SRAD and AD filters were kept same. It was also observed that by increasing the number of iterations these filters perform over smoothing which in turn destroys the fine details of ultrasound image. Enhancement methods were also evaluated on real time BUS Images and resulting images are displayed in Fig.5.

\subsection{Results}

A cumulative Matlab code was designed for different enhancement technique and values of different quality parameters were recorded. Table 1 lists the quantitative results at different noise levels for different methods. 
Table 1 SNR, EPI and SSIM values for different noise levels.

\begin{tabular}{|c|c|c|c|c|c|c|c|c|c|c|c|}
\hline \multicolumn{2}{|c|}{ Noise Level $\downarrow$} & Noisy & HE & MEAN & MEDIAN & LEE & FROST & WIENER & WAVELETS & SRAD & AD \\
\hline $\mathbf{S}$ & $\boldsymbol{\sigma}=\mathbf{0 . 3}$ & 18.19 & 1.78 & 9.06 & 9.78 & 11.81 & 13.61 & 18.20 & 16.38 & 16.87 & $\mathbf{1 9 . 6 2}$ \\
$\mathbf{N}$ & $\boldsymbol{\sigma}=\mathbf{0 . 5}$ & 13.62 & 1.53 & 8.93 & 9.56 & 11.23 & 12.61 & 16.08 & 14.56 & 15.17 & $\mathbf{1 7 . 7 3}$ \\
$\mathbf{R}$ & $\boldsymbol{\sigma}=\mathbf{0 . 7}$ & 10.74 & 1.17 & 8.78 & 9.41 & 10.72 & 11.71 & 14.14 & 12.52 & 13.74 & $\mathbf{1 5 . 4 2}$ \\
& $\mathbf{\sigma}=\mathbf{0 . 9}$ & 8.63 & 0.99 & 8.52 & 8.89 & 9.97 & 10.61 & 12.30 & 10.32 & 12.11 & $\mathbf{1 3 . 7 4}$ \\
\hline $\mathbf{E}$ & $\boldsymbol{\sigma}=\mathbf{0 . 3}$ & 0.9236 & 0.3679 & 0.0533 & 0.6221 & 0.6571 & 0.5642 & 0.9395 & 0.9133 & 0.9292 & $\mathbf{0 . 9 8 4 5}$ \\
$\mathbf{P}$ & $\boldsymbol{\sigma}=\mathbf{0 . 5}$ & 0.8191 & 0.3466 & 0.0520 & 0.6009 & 0.6633 & 0.4899 & 0.8994 & 0.7927 & 0.8767 & $\mathbf{0 . 9 5 0 8}$ \\
$\mathbf{I}$ & $\boldsymbol{\sigma}=\mathbf{0 . 7}$ & 0.7151 & 0.3362 & 0.0417 & 0.5393 & 0.6213 & 0.4405 & 0.8322 & 0.6921 & 0.8092 & $\mathbf{0 . 8 8 8 4}$ \\
& $\boldsymbol{\sigma}=\mathbf{0 . 9}$ & 0.6189 & 0.3320 & 0.0302 & 0.5337 & 0.582 & 0.3871 & 0.7523 & 0.5832 & 0.7433 & $\mathbf{0 . 7 8 4 8}$ \\
\hline $\mathbf{S}$ & $\boldsymbol{\sigma}=\mathbf{0 . 3}$ & 0.8012 & 0.2765 & 0.8124 & 0.9001 & 0.8124 & 0.8726 & 0.9128 & 0.8715 & 0.9133 & $\mathbf{0 . 9 5 0 6}$ \\
$\mathbf{S}$ & $\boldsymbol{\sigma}=\mathbf{0 . 5}$ & 0.6413 & 0.2580 & 0.7813 & 0.8547 & 0.7808 & 0.7786 & 0.8082 & 0.7969 & 0.8263 & $\mathbf{0 . 8 7 0 7}$ \\
$\mathbf{I}$ & $\boldsymbol{\sigma}=\mathbf{0 . 7}$ & 0.5264 & 0.2452 & 0.7425 & 0.8014 & 0.7069 & 0.6935 & 0.7339 & 0.7273 & 0.7390 & $\mathbf{0 . 7 8 1 4}$ \\
$\mathbf{M}$ & $\boldsymbol{\sigma}=\mathbf{0 . 9}$ & 0.4428 & 0.2263 & 0.7010 & 0.7474 & 0.6350 & 0.6136 & 0.6506 & 0.6863 & 0.6538 & $\mathbf{0 . 6 8 3 9}$ \\
\hline
\end{tabular}

\subsection{Analysis}

To demonstrate the performance comparison among different methods graphically, results are presented in Fig1 to Fig3.In these figures $\mathrm{x}$-axis represents various image enhancement methods and $y$-axis represents, values of different quantitative parameters.

"Fig1" shows that SNR values for Histogram Equalization method is very less as compared to other techniques. It proves that $\mathrm{HE}$ is not a suitable method for speckle noise reduction. All other methods used in comparison gives better SNR values whereas Anisotropic Diffusion filter gives best results. Second observation is that SNR value for all filters decreases with increase in noise but at any level Anisotropic Diffusion filter gives the best SNR value among all techniques compared in this paper.

As edges in ultrasonic images contain the most important diagnostic information, so a filter should both smooth speckle and preserve edges. "Fig.2" illustrates the graphical representation of EPI values. It represents that EPI values for MEAN filter are undesirable since LEE, Frost and Wavelets filters slightly retain the edges. Wiener, SRAD and $\mathrm{AD}$ filters gives desired results. In reference to EPI values, Anisotropic Diffusion filters exhibit best performance.

"Fig.3" represents the Structural Similarity Index Values against different techniques for different noise levels. For better quality results SSIM value should be near one which is given by diffusion based filters. SSIM values for HE method lies near zero. It proves that HE distorts the fine details and edges in BUS images. SSIM values for Wiener filter are also compatible to that of SRAD and AD filters but it need more computation.

Visual inspection of processed images in "Fig.4" reveals that diffusion based filters gives better results in terms of speckle smoothing and edge preservation. For homogeneous regions, Median, LEE, Frost and Wavelet filters perform more smoothing but at the same time they destroy some fine details. In medical ultrasonic images, fine details contains the diagnostic information so diffusion based filters can be used for enhancement of B-Mode breast ultrasound images.

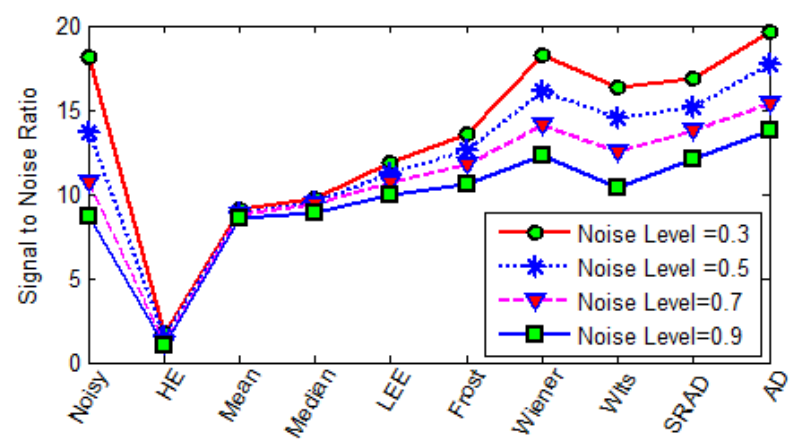

Fig. 1: Performance comparison in terms of SNR values

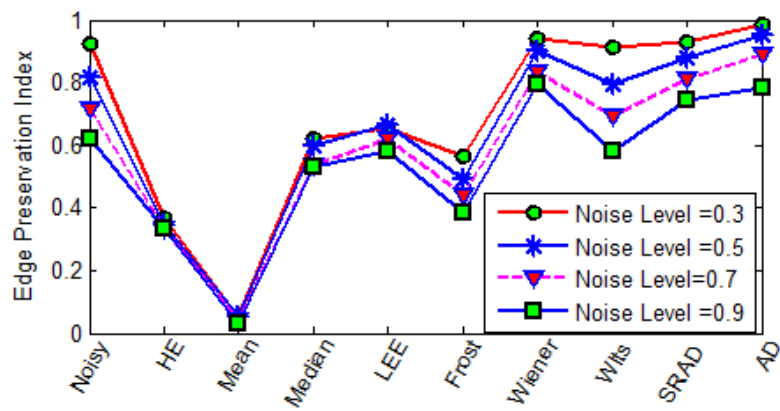

Fig. 2: Performance comparison in terms of EPI values

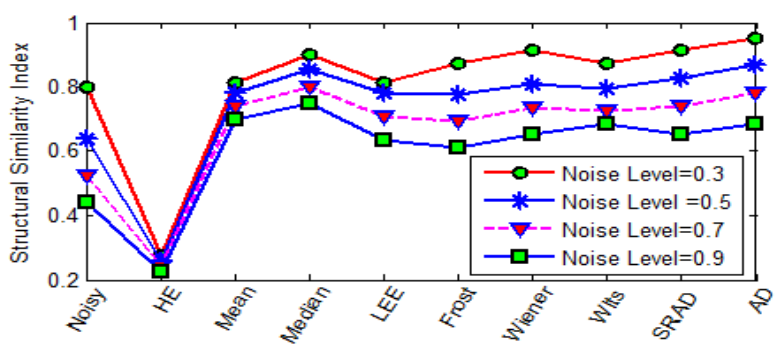

Fig. 3: Performance comparison in terms of SSIM values. 


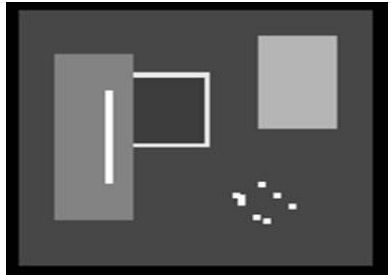

(a)

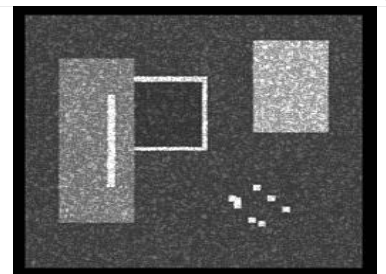

(b)

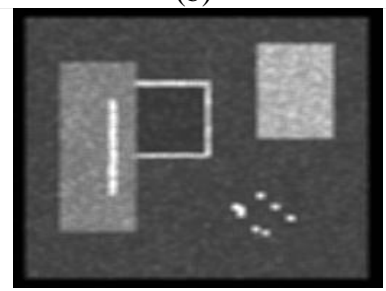

(d)

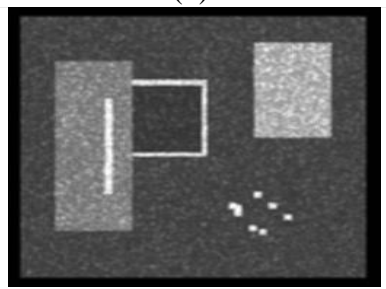

(f)

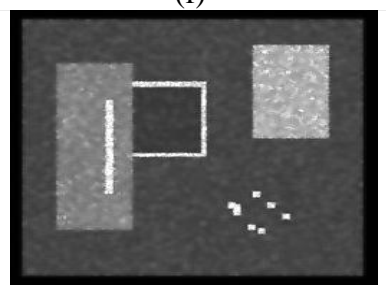

(h)

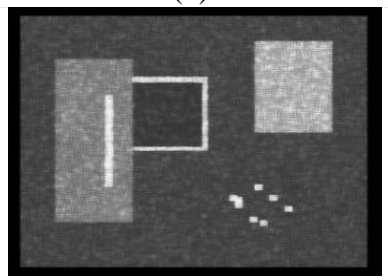

(j)

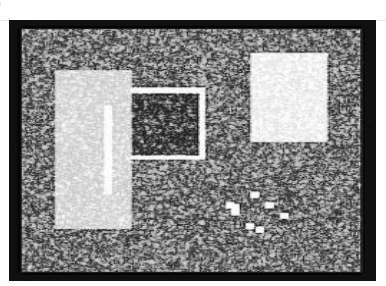

(c)

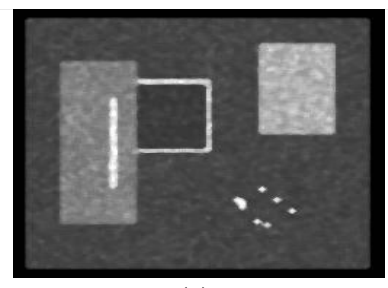

(e)

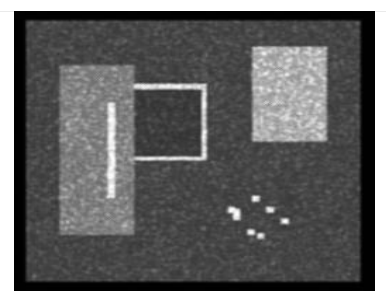

(g)

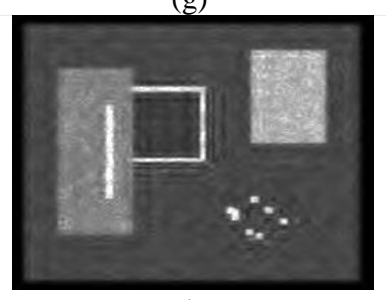

(i)

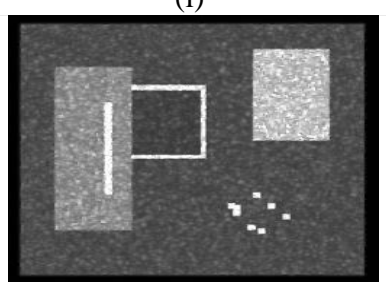

(k)
Fig 4: (a) Noise free test image [15] (b) Speckle simulated Image $(\sigma=0.7)$ (c)Histogram equiliazed image (d) Mean filters output (e) Median filters output (f) Lee filteres output (g) Frost filters output (h) Wiener filters output (i) Wavelet filters output (j) SRAD filters output (k) Anisotropic Diffusion filters output.

\section{CONCLUSION}

In this paper, performance of B-Mode breast ultrasound image enhancement techniques is tested. For performance comparison, Histogram Equalization, Mean, Median, Lee, Frost, Wiener, Wavelets, and diffusion based filters (SRAD and AD) are selected. Study shows that HE enhances the contrast of image but it cannot retain the structure and edges of original objects in input image. Mean and Median filters slightly enhances the input image. LEE, Frost and Wavelet based filters perform smoothing in homogeneous regions but these filters cannot preserve important diagnostic information. Performance metrics of Wiener filters are compatible with diffusion based filters (SRAD and AD), but it need more computation time. From quantitative and visual results, it can be concluded that Diffusion based filters outperform all other techniques. These filters smoothes homogeneous regions preserve edges and simultaneously enhance the contrast of input B-Mode breast ultrasound image. However their different numerical implementation may give different results under varied conditions.

\section{REFERENCES}

[1] K. Classic, "Medical imaging physics, fourth edition," Health Physics, vol.83, no. 6, pp. 921-921, 2002.

[2] T. L. Szabo, "Diagnostic Ultrasound Imaging," in Academic Press Series in Biomedical Engineering, Elsevier Academic Press, 2004.

[3] R. C. Gonzalez and R. E. Woods, "Digital Image Processing", $3^{\text {rd }}$ edition, Pearson Education Ins, 2008.

[4] Chen Soong-Der and Ramli, Abd.Rahman, 'Minimum Mean Brightness Error Bi Histogram Equalization in Contrast Enhancement', IEEE Transactions on Consumer Electronics, Vol.49, No.4, 2003, pp.1310-1319.

[5] T. Loupas, W. N. Mcdicken, and P. L. Allen, "An adoptive weighted median filter speckle suppression in medical ultrasound images," IEEE Trans. Circuits Sys., vol. 36, pp. 129-135, 1989.

[6] Xin-Yu Zhang, Lan Ge , Tian-Fu Wang, “ EntropyBased Local Histogram Equalization for Medical Ultrasound Image Enhancement" ,IEEE Conference on Bioinformatics and Biomedical Engineering, 16-18 May 2008. pp 2427-2429.

[7] J. S. Lee, "Digital image enhancement and noise filtering by use of local statics,"IEEE Transactions on pattern analysis and Machine Intelligence,vol.2,no.2,pp-165168,1980

[8] J. S. Lee, "Refined filtering of image noise using local statistics,"Computer Vision, Graphics and Image Processing, vol. 15, pp. 380-389, 1981.

[9] V. S. Frost, J. A. Stiles, K. S. Shanmugan, and J. C. Hltzman, "A model for radar images and its application to adoptive digital filtering for multiplicative noise," IEEE Trans. Pattern Anal. Machine Intell. , vol. PAMI-4, pp. 157-165, 1982.

[10] P. Perona and J. Malik, "Scale space and edge detection using anisotropic diffusion", IEEE Transaction on Pattern Analysis Machine Intelligence,Vol.12, pp 629639, 1990.

[11] Yongjain $\mathrm{Yu}$ and Scott.T.Acton "Speckle Reducing Anisotropic Diffusion", IEEE Transactions on image processing, Vol. 11, No, 11, 2002, pp-1260-1270.

[12] Lakhwinder Kaur , Savita Gupta , R. C. Chauhan, "Image Denoising using Wavelet Thresholding", Indian conference on Computer Vision, Graphics and Image Processing, Ahmedabad.2002. 
[13] Z. Wang and A. C. Bovik, "A universal image quality index," IEEE Signal Processing Letters, vol. 9, pp. 8184, March 2002.

[14] A. M. Eskicioglu and P. S. Fisher, "Image quality measures and their performance," IEEE Trans. on Communications, vol. 43, no. 12, pp. 2959-2965, Dec. 1995.

[15] A. Pizurika et al. "A versatile wavelet domain noise filtration technique for medical imaging", IEEE Transactions Medical Imaging, Vol. 22 No.2, 2003.

\section{APPENDIX}

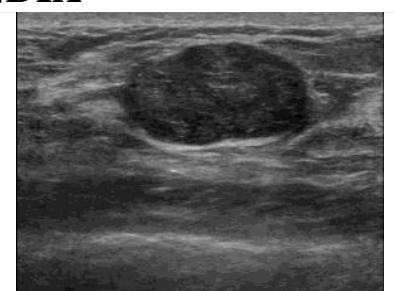

(a)

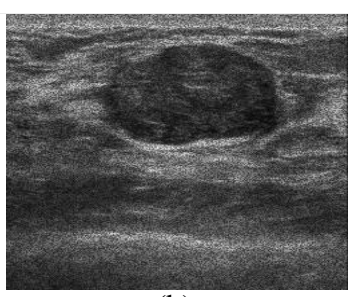

(b)

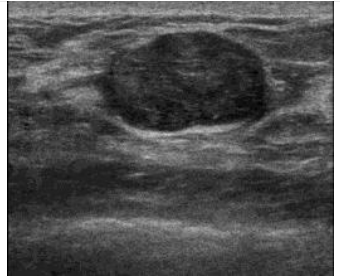

(d)

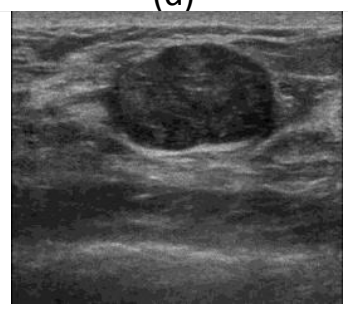

(f)

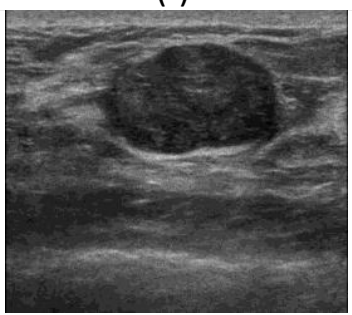

(h)

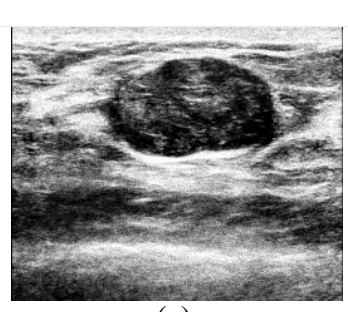

(c)

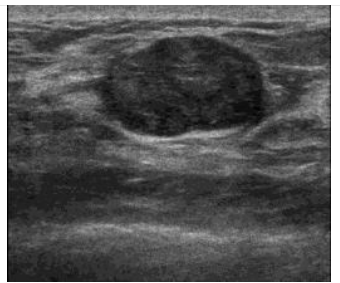

(e)

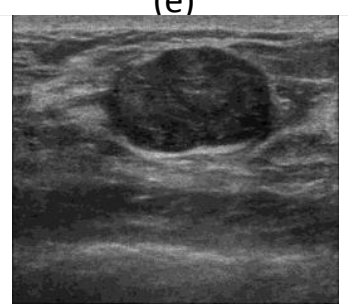

(g)

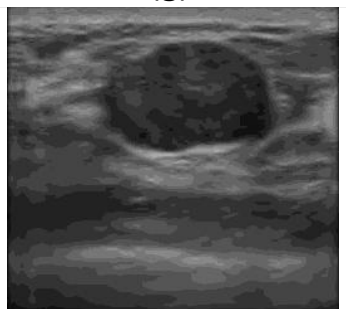

(i)
[16] A. K. Jain, "Fundamentals of Digital Image Processing", 1st ed., Prentice-Hall, Inc, 1989.

[17] S. Gupta, L. Kaur, R. C. Chauhan, and S. C. Saxena, “A versatile technique for visual enhancement of medical ultrasound image," Journal of Digital Signal Processing, Elsevier, vol. 17, pp. 542-560, January 2007.

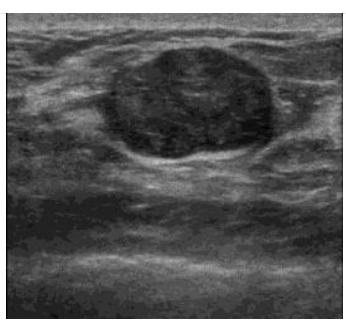

(j)

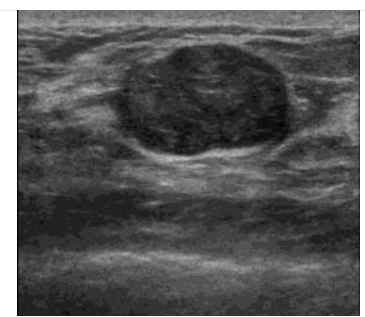

(k)
Fig 5: (a) Noise free B-Mode BUS image (b) Speckle simulated Image $(\sigma=0.3)$ (c)Histogram equiliazed image (d) Mean filters output (e) Median filters output (f) Lee filteres output (g) Frost filters output (h) Wiener filters output (i) Wavelet filters output (j) SRAD filters output (k) Anisotropic Diffusion filters output. 